\title{
Mono- and Bi-weekly Hypofractionated Radiation Therapy for the Treatment of Epithelial Skin Cancer in Very Elderly Patients
}

\author{
MAURIZIO VALERIANI ${ }^{1}$, LUCA NICOSIA ${ }^{1}$, LINDA AGOLLI $^{1}$, \\ CHIARA REVERBERI ${ }^{1}$, EMANUELA GALVAGNO ${ }^{1}$, VITALIANA DE SANCTIS ${ }^{1}$, \\ GIUSEPPE MINNITI ${ }^{1}$, MARTA CARLESIMO ${ }^{2}$ and MATTIA F. OSTI ${ }^{1}$
}

Departments of ${ }^{1}$ Radiation Oncology, and ${ }^{2}$ Dermatology, Sant' Andrea Hospital, Rome, Italy

\begin{abstract}
Background/Aim: Epithelial skin cancer frequently occurs in the elderly population, sometimes in an advanced stage, when intensive treatments are needed. Radiotherapy can achieve high response rates. We evaluated efficacy and tolerability of hypofractionated radiotherapy in a population of very elderly patients with locally advanced epithelial skin cancer. Patients and Methods: Two different hypofractionated schedules were administered (21 patients): $6 \mathrm{~Gy}$ in 10 bi-weekly fractions (13 lesions) and $5 \mathrm{~Gy}$ in 12 bi-weekly fractions (13 lesions). Median age at treatment was 88 years, life expectancy was $\leq 5$ years in $90.5 \%$. Results: The overall response rate was $96.1 \%$, with $92.4 \%$ complete responses. All patients experienced an improvement of their symptoms and a reduction of pain and medication. The median overall survival time was 28 months (95\% confidence interval=4.7-51.2 months). At the time of analysis, $52.3 \%$ of patients had died. Conclusion: Hypofractionated radiotherapy is an effective option of treatment, with low toxicity and optimal results, and can also be safely administered to these frail patients.
\end{abstract}

Epithelial skin cancer is a common neoplasm. The median patient age at diagnosis is 74 years. Its incidence is rapidly increasing probably due to the greater sun exposure of the population. The majority of the tumors are basal cell carcinomas (BCC), the remainder are squamous cell carcinomas (SCC) (1). Epithelial skin cancer is locally aggressive and the prognosis depends on stage and histology. Advanced stage and poor histology, such as SCC, adenoid or

Correspondence to: Luca Nicosia, Department of Radiation Oncology, Sant'Andrea Hospital, via di Grottarossa 1036-38, Rome, Italy. Tel: +39 0633776160, Mobile: +39 3291598888, e-mail: lucanicosia.rg@gmail.com

Key Words: Hypofractionation, elderly patients, epithelial skin cancer. adenosquamous carcinomas correlates with a higher likelihood of metastasis and poor prognosis. Nevertheless primary definitive treatment for localized tumors can be curative (2). Treatment options include surgery, radiotherapy, cryoablation, cauterization, photodynamic therapy and topical treatment with 5-fluoruracil or imiquimod cream. Surgery and radiotherapy (RT) are considered the most effective treatment options (3). RT with standard fractionation can achieve a high rate of local control (4-9).

Although screening has increased the identification of precancerous lesions, in some cases, skin cancer is still frequently diagnosed in an advanced stage and in elderly people; at this stage, intensive and sometimes disfiguring treatments are needed. Invasive or time-consuming interventions (such as conventional RT) sometimes cannot be performed or completed in elderly patients due to comorbidities, cognitive impairment, poor living conditions or the absence of a continuity of care.

RT is a curative treatment option for elderly patients or when patients are not eligible for or decline surgery; RT can also be performed in the adjuvant setting or in the case of incomplete surgical excision, when re-excision is not possible.

The standard RT regimen consists of daily sessions (5 days per week) for 4-6 weeks. Many elderly patients may have difficulties in making multiple hospital visits due to difficulties in mobilizing, comorbidities, cognitive impairment or logistical impediments. A hypofractionated regimen, where the number of fractions and the sessions per week are reduced but the dose per fraction is increased, can be more suitable for this kind of patient; moreover, some evidence suggests that a dose per fraction of more than $3 \mathrm{~Gy}$ can actually result in better local control (7-10).

We evaluated the efficacy and tolerability of hypofractionated RT in a population of very elderly patients with locally advanced-stage epithelial skin cancer. Because local treatment can be curative, our primary endpoints were local control (LC) and compliance with treatment. Secondary 
end-points were the socio-economic impact (in terms of reduction of the number of RT sessions and tumor medications, hospital visitation and nurse assistance), overall survival (OS), cancer-specific survival (CSS) and toxicity.

\section{Patients and Methods}

Patient and disease characteristics. From June 2011 to September 2015, we treated 26 epithelial skin cancers in 21 very elderly patients. Patients were eligible for the treatment if they had three or more of the following inclusion criteria: age $>80$ years, unresectable epithelial skin cancer, surgery with positive margin non-eligible for re-excision, severe comorbidities and cognitive impairment.

Nine $(43 \%)$ patients were male and $12(57 \%)$ were female. Median age at the treatment was 88 years (range $=77-100$ years), 13 (62\%) patients had an Eastern Cooperative Oncology Group Performance Status (ECOG PS) of 1-2 and eight (38\%) patients had a PS of 3-4. Patient characteristics are reported in Table I.

Tumor characteristics are summarized in Table II. The most common histology was SCC, [14 lesions (54\%)]. Nineteen (73\%) tumors were unresectable because of advanced stage or patient comorbidity, six (23\%) had positive margins (micro- or macroscopic infiltration) after surgery, and one (4\%) had macroscopic local relapse 6 months after radical surgery.

Twenty-one $(80.8 \%)$ tumors were localized in the head, four $(15.4 \%)$ in the limbs and one in the chest $(3.8 \%)$, as detailed in Table III.

The current study was carried out in accordance with the Declaration of Helsinki (1964) and the Internal Review Board approved the study. Written inform consent was obtained from patients or their legal representatives.

Treatment. Patients were treated using electron beam or photons depending on tumor site, dimensions and depth. Electron beam energy (range=6-12 MeV) was chosen on the basis of the estimated tumor depth. The target volume consisted of the tumor area plus a margin of $1-2 \mathrm{~cm}$ to include microscopic extension. The skinsparing effect of the electron beam therapy was compensated, where necessary, by bolus to the target area. Patients treated with photons underwent pre-treatment computed tomography. The gross tumor volume (GTV) was defined as the entire tumor mass; the clinical target volume (CTV) was obtained by adding a $1 \mathrm{~cm}$ margin in all directions to include the microscopic extension. A margin of $1 \mathrm{~cm}$ in all directions was added to the CTV to generate the planning target volume (PTV). RT was delivered with a Varian Linear Accelerator using 6-MV photon beam.

At the beginning of the study, five lesions (19.2\%) were treated with a one fraction per week schedule with incremental dose per fraction from 5 Gy for 12 fractions (three patients) to 6 Gy for 10 fractions (two patients) [biological equivalent dose (BED) range $=90-96$ Gy with $\alpha / \beta=10$ for early responding tissues].

These fractionations were chosen to give a higher BED with respect to the $66 \mathrm{~Gy}$ delivered in 30 fractions of $2.2 \mathrm{~Gy}$ (BED $80.5 \mathrm{~Gy})$ used in compliant patients at our Institution, also considering a longer total treatment time (10-12 weeks vs. 6 weeks). Subsequently, considering the good compliance of treated patients and with the aim of reducing the total treatment time, we increased the number of fractions to two per week. Two different hypofractionated schedules were administered: the first 10 cases
Table I. Patient's characteristics $(n=21)$.

\begin{tabular}{lc}
\hline Characteristic & Value \\
\hline Mean age (range) pre-RT, years & $88(77-100)$ \\
Mean age (range) at diagnosis, years & $84(66-95)$ \\
Gender, n (\%) & \\
Male & $9(43)$ \\
Female & $12(57)$ \\
ECOG PS, n (\%) & \\
1 & $4(19)$ \\
2 & $9(43)$ \\
3 & $5(24)$ \\
4 & $3(14)$ \\
Life expectancy, n (\%) & $2(9.5)$ \\
$>5$ Years & $19(90.5)$ \\
$<5$ Years & \\
Comorbidities (per type), n (\%) & $12(57)$ \\
Cardiovascular & $7(33)$ \\
Cerebrovascular & $5(24)$ \\
Metabolic & $4(19)$ \\
Osteoarticolar & $4(19)$ \\
Oncological & $2(9.5)$ \\
Pulmonary & \\
Comorbidities (per patient), n (\%) & $9(43)$ \\
$0-1$ & $6(28.5)$ \\
2 & $6(28.5)$ \\
$\geq 3$ & $8(6-12)$ \\
Charlson Age-Comorbidity Index (range) & $19.37(6.38-19.37)$ \\
Relative risk of death $(95 \%$ CI) & \\
Mobilization & $8(38)$ \\
Autonomous & $5(24)$ \\
Not-autonomous & $8(38)$ \\
Use of device & $19(90.5)$ \\
Feeding & $2(9.5)$ \\
Oral & \\
Parenteral & \\
Home status & \\
Living at home & \\
Hospice/retirement home & \\
\hline & \\
\hline
\end{tabular}

CI: Confidence interval; ECOG PS: Eastern Cooperative Oncology Group Performance status; RT: radiotherapy.

(38.5\%) were treated with 12 fractions of 5 Gy and the second 11 cases $(42.3 \%)$ with 10 fractions of $6 \mathrm{~Gy}$.

We used linear-quadratic (LQ) modeling in order to equate these hypofractionation schedules to the normalized total dose (NTD) if delivered in 2-Gy fractions (12). Thus, NTD represents the dose given in 2-Gy fractions that would have an equivalent biologic effect as the new hypofractionated dose:

$N T D=[$ Dnew $\times(1+$ dnew $/ \alpha / \beta)] /(1+2 / \alpha / \beta)$

where Dnew and dnew were the total dose and dose per fraction for a suggested hypofractionation scheme, respectively. The total dose of $60 \mathrm{~Gy}$ given in 12 or 10 fractions, yielded NTD3 of 96 and 108 Gy $\left(\alpha / \beta=3\right.$ Gy for late toxicity) and $\mathrm{NTD}_{10}$ of 75 and $80 \mathrm{~Gy}$ $(\alpha / \beta=10$ Gy for acute toxicity), respectively. The NTD for each schedule and treatment details are reported in Table IV. 
Table II. Tumor characteristics $(n=26)$.

\begin{tabular}{lc}
\hline Characteristic & No. of tumors $(\%)$ \\
\hline Histology & \\
SCC & $14(54)$ \\
BCC & $6(23)$ \\
Basal/squamous & $2(7.5)$ \\
Other & $3(11.5)$ \\
Unknown & $1(4)$ \\
Stage & \\
I & $1(4)$ \\
II & $17(65)$ \\
III & $2(8)$ \\
IV & $6(23)$ \\
Previous surgery & \\
Yes & $7(27)$ \\
No & $19(73)$ \\
Relapse after R0 surgery & \\
R Status & $1(4)$ \\
R1 & $2(8)$ \\
R+ & $4(15.5)$ \\
\hline
\end{tabular}

SCC: Squamous cell carcinoma; BCC: basal cell carcinoma; R0: negative surgical margins; R1: microscopical tumor infiltration; $\mathrm{R}+$ macroscopical tumor infiltration.

Follow-up. Acute and late toxicity were graded according to the European Organisation for Research and Treatment of Cancer/Radiation Therapy Oncology Group criteria (13). Clinical evaluation was performed weekly during the course of RT, 1 month after the end of RT and thereafter every 3 months for 2 years. Each visitation included evaluation by the radiation oncologist and the dermatologist.

Statistical method. The analysis of the tumor response and LC was made per tumor, because a single patient could have multiple tumors. LC, OS and CSS were calculated from the end of the RT. OS was defined as the time from the end of RT to the last followup or death.

Kaplan-Meier method was used for calculating OS and CSS and factors that affect LC, and a Chi-square test was used in the univariate analyses. Data were prospectively collected, according to our internal protocol, and a retrospective statistical analysis was conducted using SPSS 16.0 software (SPSS Inc,, Chicago, IL, USA).

\section{Results}

Response to treatment, local and distant recurrence. Overall, the response rate was $96.1 \%$ (25/26 lesions), of these 24 $(92.4 \%)$ had a complete response and one $(3.8 \%)$ a partial response. One case $(3.8 \%)$ was suspicious for progression but biopsy could not be performed because the patient died of kidney failure 5 months after RT completion, therefore we considered this case as a progression. Two (9.5\%) patients had distant progression: the first patient had an SCC of the leg with locoregional nodal involvement at diagnosis and developed distant metastases 2 months after the end of the RT; the other
Table III. Tumor localization $(n=26)$.

\begin{tabular}{ll}
\hline Location & $\mathrm{n}(\%)$ \\
\hline Cheek & $6(23)$ \\
Scalp & $4(15.3)$ \\
Nose & $3(11.6)$ \\
Temple & $3(11.6)$ \\
Inferior limb & $3(11.6)$ \\
Forehead & $2(7.6)$ \\
Ear & $2(7.6)$ \\
Superior limb & $1(3.9)$ \\
Trunk & $1(3.9)$ \\
Eyelid & $1(3.9)$ \\
\hline
\end{tabular}

one had an advanced SCC of the scalp and developed a metastasis in a single locoregional node 13 months after RT.

Toxicity, compliance with treatment and quality of life. Sixteen (61.5\%) cases developed acute toxicity, 12 (46.2\%) of these had grade 1-2 toxicity and four (15.3\%) grade 3. Late toxicity, represented by necrosis, occurred in three $(11.5 \%)$ cases, but none needed surgical intervention. Compliance of patients was good; only three $(11.5 \%)$ treatments were interrupted and two $(7.6 \%)$ of these were afterwards not completed due to severe toxicity: the first case had grade 3 mucositis and dysphagia, interrupting the treatment at the seventh fraction (total dose $=42 \mathrm{~Gy}$ ) and died 4 months later due to myocardial infarction. The second patient was treated for a tumor of the nose and treatment was interrupted for 2 months due to infectious pneumonitis; after resumption, the therapy was definitively ended at the ninth of 10 fractions due to severe pain and conjunctivitis. Cosmetic assessment was not performed.

All patients experienced an improvement of their symptoms, with a reduction of pain. All patients with stage III/IV lesions and $61.1 \%$ (11 patients) of patients with stage II tumors received daily, sometimes multiple, medications since before RT, in most cases administered by a private nurse at home, the cost of which was borne by the family, in some other cases medications were given by family members trained to do so. We observed a reduction in the number of medications during the course of RT proportional to the improvement of the lesion. At the first follow-up, 23 (88.4\%) lesions had completely disappeared and no medications were needed, only the three patients with necrosis required further medication, but no surgical intervention.

Survival and treatment of recurrence. Median follow-up was 7 months (range $=4-40$ months). The median OS in our series was 28 months (95\% confidence interval=4.7-51.2). Sixmonth survival was $63.8 \%, 1$ - and 2-year OS were both 
Table IV. Treatment characteristics $(n=26)$.

\begin{tabular}{lcccccc}
\hline Total dose & Dose/fr & No. of fractions & Schedule & NTD $_{3 G y}{ }^{*}$ & NTD $_{10 \mathrm{~Gy}}{ }^{* *}$ & BED $_{10 \mathrm{~Gy}}$ \\
\hline $60 \mathrm{~Gy}(\mathrm{n}=3)$ & $5 \mathrm{~Gy}$ & 12 & Mono-weekly & $96 \mathrm{~Gy}$ & $75 \mathrm{~Gy}$ & $90 \mathrm{~Gy}$ \\
$60 \mathrm{~Gy}(\mathrm{n}=2)$ & $6 \mathrm{~Gy}$ & 10 & Mono-weekly & $108 \mathrm{~Gy}$ & $80 \mathrm{~Gy}$ & $96 \mathrm{~Gy}$ \\
$60 \mathrm{~Gy}(\mathrm{n}=10)$ & $5 \mathrm{~Gy}$ & 12 & Bi-weekly & $96 \mathrm{~Gy}$ & $75 \mathrm{~Gy}$ & $90 \mathrm{~Gy}$ \\
$60 \mathrm{~Gy}(\mathrm{n}=11)$ & $6 \mathrm{~Gy}$ & 10 & Bi-weekly & $108 \mathrm{~Gy}$ & $80 \mathrm{~Gy}$ & $96 \mathrm{~Gy}$ \\
\hline
\end{tabular}

NTD: Normalized total dose; *calculated with an $\alpha / \beta=3$ Gy for low-proliferating tumors and for late toxicity; **calculated with an $\alpha / \beta=10 \mathrm{~Gy}$ for early-responding tissues; BED: biological equivalent dose calculated with an $\alpha / \beta=10$ Gy for early-responding tissues.

$51.2 \%$. At the time of analysis, $11 / 21(52.3 \%)$ patients had dead: one $(4.7 \%)$ due to systemic spread that occurred 2 months after RT completion, one $(4.7 \%)$ with suspicion of local progression died of kidney failure 5 months after treatment; the other nine $(42.7 \%)$ patients died from causes not related to the tumor. Ten patients $(47.6 \%)$ were alive at the time of analysis: one $(4.7 \%)$ out of these patients had developed a metastasis in a locoregional node and received an RT course of $6 \mathrm{~Gy}$ in 10 fractions twice per week.

The median CSS was not reached. One and 2-year CSS were both $95 \%$.

Univariate analysis. No differences were seen in response to treatment by histological type (SCC vs. non-SCC; $p=1$.); nevertheless, it should be noted that the only partial response and the suspected case of progression both occurred in the SCC group. Distant recurrence occurred in two $(9.5 \%)$ patients, both in the SCC group, but correlations with response and with fractionation were not statistically significant.

No differences were seen in response to treatment according to factors such as sex, age, life expectancy, pre-treatment PS, localization of tumor, staging, prior surgery and treatment interruption. Moreover, the two different fractionations (5 Gy/12 fr and $6 \mathrm{~Gy} / 10 \mathrm{fr}$ ) and the administration schedule (mono- or biweekly) did not significantly correlate with response, histology or toxicity.

\section{Discussion}

RT is a mainstay in the treatment of epithelial skin cancer and is the most effective option for patients not suitable for surgery. LC rates after RT with standard fractionation for primary BCC and SCC have been reported to be $87-98 \%$ and $56-97 \%$, respectively (4-8). Some evidence showed that hypofractionated schedules could achieve a high rate of LC (10). Elderly patients may not be able to attend hospital daily because of comorbidities or poor socio-economic conditions, therefore they can benefit from a reduction of the number of fractions. The rationale of the present study comes from the need to administer a safe and effective treatment to patients that are unable to comply with daily treatment, such as elderly ones, patients with comorbidities or cognitive impairment, and to reduce the cost of treatments.

The primary end-points were LC and compliance with treatment; secondary end-points were the socio-economic impact (in terms of reduction of the number of RT sessions and tumor medications, hospital visitation andnurse assistance), OS, CSS and toxicity. The altered fractionation well suited this objective.

The first evidence on the efficacy of hypofractionation for these tumor types comes from a study of Locke et al. (10); the study showed that the rate of LC for BCC of $1.1-5 \mathrm{~cm}$ was higher when the dose per fraction increased from $<2$ to 3.01 to $4 \mathrm{~Gy}(75 \%, 95 \%$ and $100 \%$ respectively; $p=0.01)$. Moreover, patients receiving a total dose $>60$ Gy had significantly lower LC when a fraction size $<2$ Gy was used compared with a larger fraction size $(p=0.01)$. An improvement in local control was also seen for SCC when the dose per fraction increased $(73 \%$ for $<2$ Gy to $100 \%$ for $>4$ Gy in $\leq 1 \mathrm{~cm}$ lesions), even if the association was not statically correlated.

In a study of van Hezewijk et al. the Authors administered two hypofractionation schedules (54 Gy in 18 fractions of $3 \mathrm{~Gy}$ or $44 \mathrm{~Gy}$ in 10 fractions of $4.4 \mathrm{~Gy}$ ). The increased dose per fraction resulted in a higher local recurrence-free rate, increasing from $96.1 \%$ in the 54 Gy schedule to $97.5 \%$ in the 44 Gy schedule (9). Nevertheless the rate of T1-2 lesions, which are known to be the best responders to local treatment, was quite high $(86.8 \%)$ compared with our series.

LC in the present study was $96.1 \%$ (25 out of 26 treated lesions), with only ase suspicious for progression; the complete rate was $92.4 \%$ ( 24 out of 26 treated lesions). The rate of stage III or IV tumors $(31 \%)$ of our study population was high in comparison with other studies on curative hypofractionation $(9,10)$ or adjuvant hypofractionation $(15)$.

From the analysis of the literature available to date, some consideration about the total dose of the treatment should be made. As previously reported (8), the association of increasing BED with improved LC supports a radiation dose-response relationship. 
Khan et al. administered two regimens of $40 \mathrm{~Gy}$ in 10 fractions and $50 \mathrm{~Gy}$ in 20 fractions (BED range $=56-62.5$ with $\alpha / \beta=10$; NTD range $=46.7-52.1$ Gy with $\alpha / \beta=10$ ); they reported an LC rate of $84.2 \%$ (14), which is lower than those of other studies, concluding that an NTD higher than $56 \mathrm{~Gy}$ is recommended, especially for lesions greater than $2 \mathrm{~cm}$. The higher BED reported in the study of van Hezewijk et al. (9) (range=63.4-70.2 Gy, with $\alpha / \beta=10$ ) resulted in higher LC (96.1-97.5\%), with a crude rate of recurrence of $3.4 \%$; nevertheless considering lesions per stage, the rate of recurrence for T3 tumors was significantly higher $(18.1 \%, 2 / 11$ lesions) and both events occurred in the 44 Gy group. The BED of our treatments was higher than the that reported in the literature (BED range $=90-96 \mathrm{~Gy}$ ), with only one case $(4.7 \%)$ suspicious for local progression and two cases $(9.5 \%)$ out of 21 patients with distant metastases. Both distant recurrences occurred in patients with very advanced SCC: the first had nodal involvement at the time of local treatment and died of metastatic spread a few months after the end of the therapy, probably reflecting an early dissemination of the disease; the second had a T4 tumor of the ear and developed a single metastasis in a locoregional node that has been treated with a $6 \mathrm{~Gy} \times 10$ biweekly RT. After a median OS of 28 months, we reported no cases of local recurrence, but only one suspicious of progression; this result, even if retrospective, is remarkable, considering that the median time to local relapse for SCC is 3.3-5 months and for BCC is 10.4-40.5 months $(4,9,16)$.

With regard to histology, it is known that SCC is locally and distantly aggressive. We reported one case suspicious for local progression and two cases of distant progression occurring in patients affected by SCC, but the correlation with the treatment schedule was not statistically significant. This result probably derived from the low rate of events in our series, therefore by increasing the series size, it should be possible to observe a significant difference.

The population included in the present study consisted of a homogeneous series of elderly patients with advancedstage skin tumors who were unable to undergo surgery, nor daily RT, in most cases they also had comorbidities or cognitive impairment. For these patients, the reduction of the number of fraction resulted in a higher adherence to treatment; we reported only three $(11.5 \%)$ cases of interruption, but only in one case was the reason due to unacceptable toxicity and the treatment was then not completed. The toxicity rate reported in our series suggests the safety of the proposed schedules.

Limitations of our study are the low sample size and its retrospective nature which does not allow us to compare the relative effectiveness of the proposed treatments, even considering the low rate of recurrence. Prospective studies are needed to confirm these data and to evaluate efficacy also in the general population.

\section{Conclusion}

Hypofractionated RT is an effective treatment option and can be safely administered in elderly patients, with low toxicity and optimal results. The toxicity is acceptable and the rate of withdrawal is low. Considering the high rate of LC achieved with both schedules, we suggest the use of the schedule of 6 Gy in 10 (biweekly) fractions in this setting of elderly patients, mainly due to the shorter duration of the course and in the view of more sustainable treatment administration for these frail patients and in order to reduce costs.

\section{References}

1 Lomas A, Leonardi-Bee $\mathrm{J}$ and Bath-Hextall $\mathrm{F}$ : A systematic review of worldwide incidence of nonmelanoma skin cancer. $\mathrm{Br}$ J Dermatol 166(5): 1069-1080, 2012.

2 McCusker $M$, Basset-Seguin N, Dummer R, Lewis $K$, Schadendorf D, Sekulic A, Hou J, Wang L, Yue H and Hauschild A: Metastatic basal cell carcinoma: Prognosis dependent on anatomic site and spread of disease. Eur J Cancer 50(4): 774783, 2014.

3 Bath-Hextall FJ, Perkins W, Bong $J$ and Williams HC: Interventions for basal cell carcinoma of the skin. Cochrane Database Syst Rev 24(1): CD003412, 2007.

4 Kwan W, Wilson D and Moravan V: Radiotherapy for locally advanced basal cell and squamous cell carcinomas of the skin. Int J Radiat Oncol Biol Phys 60(2): 406-411, 2004.

5 Childers BJ, Goldwyn RM, Ramos D, Chaffey J and Harris JR: Long-term results of irradiation for basal cell carcinoma of the skin of the nose. Plast Reconstr Surg 93(6): 1169-1173, 1994.

6 Rowe DE, Carroll RJ and Day CL Jr.: Prognostic factors for local recurrence, metastasis, and survival rates in squamous cell carcinoma of the skin, ear, and lip. Implications for treatment modality selection. J Am Acad Dermatol 26(6): 976990, 1992.

7 Tsao MN, Tsang RW, Liu FF, Panzarella T and Rotstein L: Radiotherapy management for squamous cell carcinoma of the nasal skin: the Princess Margaret Hospital experience. Int $\mathbf{J}$ Radiat Oncol Biol Phys 52(4): 973-979, 2002.

8 Silva JJ, Tsang RW, Panzarella T, Levin W and Wells W: Results of radiotherapy for epithelial skin cancer of the pinna: the Princess Margaret Hospital experience, 1982-1993. Int J Radiat Oncol Biol Phys 47(2): 451-459, 2000.

9 van Hezewijk M, Creutzberg CL, Putter H, Chin A, Schneider I, Hoogeveen M, Willemze R and Marijnen CA: Efficacy of a hypofractionated schedule in electron beam radiotherapy for epithelial skin cancer: analysis of 434 cases. Radiother Oncol 95(2): 245-249, 2010.

10 Locke J, Karimpour S, Young G, Lockett MA and Perez CA: Radiotherapy for epithelial skin cancer. Int J Radiat Oncol Biol Phys 51(3): 748-755, 2001.

11 Charlson M, Szatrowski TP, Peterson J and Gold J: Validation of a combined comorbidity index. J Clin Epidemiol 47(11): 1245-1251, 1994.

12 Fowler JF: The linear-quadratic formula and progress in fractionated radiotherapy. Br J Radiol 63(740): 679-694, 1989. 
13 Cox JD, Stenz J and Pajak TF: Toxicity criteria of the radiation therapy oncology group (RTOG) and the European organization for research and treatment of cancer. Int J Radiat Oncol Biol Phys 31(5): 1341-1346, 1995.

14 Khan L, Breen D, Zhang L, Balogh J, Czarnota G, Lee J, Tsao $\mathrm{MN}$ and Barnes EA: Predictors of recurrence after radiotherapy for non-melanoma skin cancer. Curr Oncol 21(2): e326-329, 2014.

15 Kouloulias V, Papadavid E, Mosa E, Platoni K, Papadopoulos O, Rigopoulos D, Georgakopoulos J, Beli I, Karantonis F, Castana O, Dalamaga M and Kelekis N: A new hypofractionated schedule of weekly irradiation for basal cell carcinoma of the head and neck skin area in elderly patients. Dermatologic Therapy 27(3): 127-30, 2014.

16 Vargo N: Basal cell and squamous cell carcinoma. Semin Oncol Nurs 19(1): 12-21, 2003.

Received October 14, 2016

Revised December 16, 2016

Accepted December 20, 2016 